\title{
Deterministic Computation Towards Indeterminism
}

\author{
Bogdanov A.V., Gevorkyan A.S., Stankova E.N., Pavlova M.I. \\ Institute for High-Performance Computing and Information Systems \\ Fontanka emb. 6, 194291, St-Petersburg, Russia \\ bogdanov@hm.csa.ru, ashot@fn.csa.ru, lena@fn.csa.ru, meri@fn.csa.ru
}

\begin{abstract}
In the present work we propose some interpretation of the results of the direct simulation of quantum chaos.
\end{abstract}

\section{Introduction}

At the early stage of quantum mechanics development, Albert Einstein has written a work in which the question, which has become a focus of physicians attention several decades later, was touched upon. The question was: what will the classic chaotic system become in terms of quantum mechanics. He has particularly set apart the three-body system.

In an effort to realize the problem and get closer to its chaos solution in essential quantum area, M. Gutzwiller, a well known physician, have conditionally subdivided all the existing knowledge in physics into three areas [1]:

1) elementary classical mechanics, which only allows for simple regular system behaviour (regular classical area $R$ );

2) classical chaotic dynamic systems of Poincare systems ( $P$ area);

3) regular quantum mechanics, which interpretations are being considered during last 80 years $(Q$ area).

The above areas are connected by a separate bounds. Thus, Bor's correspondence principal works between $R$ and $Q$ areas, transferring quantum mechanics into classical Newton's mechanics within the limit $\hbar \rightarrow 0$. $Q$ and $P$ areas are connected by Kolmogorov's - Arnold's - Mozer's theorem (KAM). Let's note that KAM theorem allows to determine the excitations, which cause the chaotic behaviour of regular systems. Inspite of well known work by $\mathrm{Wu}$ and Parisi [2], which allows to describe $Q$-systems with the help of $P$-systems in thermodynamic limit under certain circumstances, the general principle connecting $P$ and $Q$ is not yet determined. Assuming the existence of the fourth area - quantum chaos area $Q_{c h}$, M. Gutzwiller adds that it rather serves for the puzzle description than for a good problem formulation. It is evident that the task formulated correctly in $Q_{c h}$ area is a most general one and must transfer to the abovementioned areas in its limits.

The problem of quantum chaos was studied as the example of quantum multichannel scattering in collinear three-body system [3,4]. It was shown than this task can be transformed into a problem of unharmonic oscillator with non-trivial 
time (internal time). Let's note, that in a model considered internal time is determined by a system of two non-linear differential equations of the second order. In [5] this problem was studied on the example of chemical reaction and in [6] it was applied to surface scattering. The ab initio computation even of the simple three-body systems is a challenge for some generation of computational physicists, so some new approach was proposed in [7] and beautiful example of distributed computation was demonstrated in [8].

In the present work we propose some interpretation of the results, obtained in $[7,8]$, and thus give our view of quantum chaos origination.

\section{Formulation of the problem}

The quantum multichannel scattering in the framework of collinear model is realized accordingly to follow scheme:

$$
A+(B C)_{n} \rightarrow\left\{\begin{array}{l}
A+(B C)_{m} \\
(A B)_{m}+C \\
A+B+C \\
(A B C)^{*} \rightarrow\left\{\begin{array}{l}
A+(B C)_{m} \\
(A B)_{m}+C \\
A+B+C
\end{array}\right.
\end{array}\right.
$$

with $m$ and $n$ being the vibrational quantum numbers correspondingly in (in) and (out) scattering channels. As it was shown elsewhere [3,4] the problem of quantum evolution (1) can be strictly formulated as a motion of image point with a reduced mass $\mu_{0}$ over the manyfold $M$, that is stratificated Lagrange surface $S_{p}$, in the moving over $S_{p}$ local coordinate system. In our case there is standard definition of the surface $S_{p}$

$$
\begin{gathered}
S_{p}=\left\{x^{1}, x^{2} ; 2 \mu_{0}\left(E-V\left(x^{1}, x^{2}\right)\right)>0\right\}, \\
\mu_{0}=\left\{\frac{m_{A} m_{B} m_{C}}{m_{A}+m_{B}+m_{C}}\right\}^{1 / 2},
\end{gathered}
$$

where $m_{A}, m_{B}, m_{C}$ being the masses of corresponding particles, $E$ and $V\left(x^{1}, x^{2}\right)$ being correspondingly the total energy and interaction potential of the system. The metric on the surface $S_{p}$ in our case is introduced in the following way

$$
\begin{gathered}
g_{i k}=P_{0}^{2}\left(x^{1}, x^{2}\right) \delta_{i k}, \\
P_{0}^{2}\left(x^{1}, x^{2}\right)=2 \mu_{0}\left(E-V\left(x^{1}, x^{2}\right)\right) .
\end{gathered}
$$

As to the motion of the local coordinate system, it is determined by the projection of the image point motion over the extremal ray $\Im_{\text {ext }}$ of the Lagrange manyfold $S_{p}$. Note, that for scattering problem (1) there are two extremal rays on a surface $S_{p}$ : one is connecting the $(i n)$ channel with the (out) channel of particle 
rearrangement and the other is connecting the $($ in $)$ channel with the (out) channel, where all three particles are free. ¿From now on we shall study only the case of rearrangement final channel. Let us introduce curvilinear coordinates $\left(x^{1}, x^{2}\right)$ in Euclidean space $R^{2}$ along the projection of the rearrangement extremal ray $\bar{\Im}_{\text {ext }}$ in a such way, that $x^{1}$ is changing along $\bar{\Im}_{\text {ext }}$ and $x^{2}$ is changing in orthogonal direction. In such a case the trajectory of image point is determined by the following system of the second order differential equations:

$$
x_{; s s}^{k}+\{\}_{k i j} x_{; s}^{i} x_{; s}^{j}=0 \quad(i, j, k=1,2)
$$

where \{\}$_{k i j}=(1 / 2) g^{k l}\left(g_{l j ; i}+g_{i l ; j}-g_{i j ; l}\right), g_{i j ; k}=\partial_{x^{k}} g_{i j}$.

As to the law of local coordinate system motion, it is given by the solution $x^{1}(s)$. Based on this solution the quantum evolution of the system on the manyfold $M$ is determined by the equation (see [4])

$$
\left\{\hbar^{2} \Delta_{\left(x^{1}(s), x^{2}\right)}+P_{0}^{2}\left(x^{1}(s), x^{2}\right)\right\} \Psi=0,
$$

with the operator $\Delta_{\left(x^{1}(s), x^{2}\right)}$ of the form

$$
\Delta_{\left(x^{1}(s), x^{2}\right)}=\gamma^{-\frac{1}{2}}\left\{\partial_{x^{1}(s)}\left[\gamma^{i j} \gamma^{\frac{1}{2}} \partial_{x^{1}(s)}\right]+\partial_{x^{2}}\left[\gamma^{i j} \gamma^{\frac{1}{2}} \partial_{x^{2}}\right]\right\}
$$

As to the metric tensor of the manyfold $M$, it has the following form [4]:

$$
\begin{gathered}
\gamma_{11}=\left(1+\frac{\lambda\left(x^{1}(s)\right)}{\rho_{1}\left(x^{1}(s)\right)}\right)^{2}, \quad \gamma_{12}=\gamma_{21}=0 \\
\gamma_{22}=\left(1+\frac{x^{2}}{\rho_{2}\left(x^{1}(s)\right)}\right)^{2}, \quad \gamma=\gamma_{11} \gamma_{22}>0,
\end{gathered}
$$

with $\lambda$ being de Broglie wave length on $\Im_{\text {ext }}$ and $\rho_{1}, \rho_{2}$ being the principle curvatures of the surface $S_{p}$ in the point $x^{1} \in \Im$ in the directions of coordinates $x^{1}, x^{2}$ changes

$$
\begin{gathered}
\rho_{1}=\frac{P_{0}\left(x^{1}(s), 0\right)}{P_{0 ; x^{1}}\left(x^{1}(s), 0\right)}, \quad \rho_{2}=\frac{P_{0}\left(x^{1}(s), 0\right)}{P_{0 ; x^{2}}\left(x^{1}(s), 0\right)}, \\
\lambda=\frac{\hbar}{P_{0}\left(x^{1}(s), 0\right)}, \quad P_{0 ; x^{i}}=\frac{d P\left(x^{1}(s), x^{2}\right)}{d x^{i}} .
\end{gathered}
$$

Note, that the main difference of (5) from Schrödinger equation comes from the fact, that one of the independent coordinates $x^{1}(s)$ is the solution of nonlinear difference equations system and so is not a natural parameter of our system and can in certain situations be a chaotic function. 


\section{Reduction of the scattering problem to the problem of quantum harmonic oscillator with internal time}

Let us make a coordinate transformations in Eq.(5):

$$
\begin{gathered}
\tau=\left(E_{k}^{i}\right)^{-1} \int_{0}^{x^{1}(s)} P\left(x^{1}, 0\right) \sqrt{\gamma} d x^{1}, \\
z=\left(\hbar E_{k}^{i}\right)^{-\frac{1}{2}} P\left(x^{1}, 0\right) x^{2},
\end{gathered}
$$

with $E_{k}^{i}$ being the kinetic energy of particle $A$ in the $(i n)$ channel, the function $P\left(x^{1}, x^{2}\right)=\sqrt{2 \mu_{0}\left[E_{k}^{i}-V\left(x^{1}, x^{2}\right)\right]}$ and with image point on the curve $\Im_{e x t}$ it is just the momentum of image point.

By expanding of $P\left(x^{1}, x^{2}\right)$ over the coordinate $x^{2}$ up to the second order we can reduce the scattering equation (5) to the problem of quantum harmonic oscillator with variable frequency in the external field, depending on internal time $\tau\left(x^{1}, x^{2}\right)$. E.g. in the case of zero external field the exact wave function of the system without some constant phase, unimportant for the expression of the scattering matrix, is of the form

$$
\begin{gathered}
\Psi^{+}(n ; \tau)=\left[\frac{\left(\Omega_{i n} / \pi\right)^{1 / 2}}{2^{n} n !|\xi|}\right]^{\frac{1}{2}} \times \\
\exp \left\{\frac{E_{k}^{i} \tau}{\hbar}-\left(n+\frac{1}{2}\right) \Omega_{i n} \int_{-\infty}^{\tau} \frac{d \tau^{\prime}}{|\xi|^{2}}+\right. \\
\left.+\frac{1}{2} \dot{\xi} \xi^{-1} z^{2}-\frac{1}{2} \dot{p} p^{-1} z^{2}\right\} H_{n}\left(\frac{\sqrt{\Omega_{i n}}}{|\xi|} z\right), \\
\dot{\xi}=d_{\tau} \xi, \quad \dot{p}=d_{\tau} p, \quad p\left(x^{1}(s)\right)=P\left(x^{1}(s), 0\right),
\end{gathered}
$$

with the function $\xi(\tau)$ being the solution of the classical oscillator equation

$$
\begin{gathered}
\ddot{\xi}+\Omega^{2}(\tau) \xi=0, \\
\Omega^{2}(\tau)=-\left(\frac{E_{k}^{i}}{p}\right)^{2}\left\{\frac{1}{\rho_{2}^{2}}+\sum_{k=1}^{2}\left[\frac{p_{; k k}}{p}+\left(\frac{p_{; k}}{p}\right)^{2}\right]\right\}, \\
p_{; k}=\frac{d p}{d x^{k}}
\end{gathered}
$$

with asymptotic conditions 


$$
\begin{gathered}
\xi(\tau) \underset{\tau \rightarrow-\infty}{\stackrel{\sim}{\longrightarrow}} \exp \left(i \Omega_{\text {in }} \tau\right), \\
\xi(\tau) \underset{\tau \rightarrow+\infty}{\stackrel{\sim}{\longrightarrow}} C_{1} \exp \left(i \Omega_{\text {in }} \tau\right)-C_{2} \exp \left(i \Omega_{\text {out }} \tau\right) .
\end{gathered}
$$

Note, that internal time $\tau$ is directly determined by the solution of $x^{1}(s)$ and therefore includes all peculiarities if $x^{1}$ behaviour.

The transition probability for that case is of the form [3,4]:

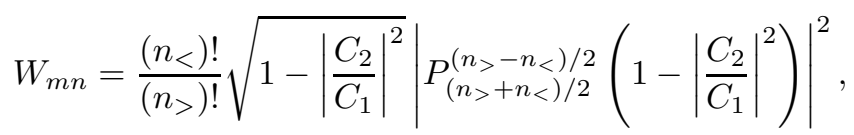

where $n_{<}=\min (m, n), n_{>}=\max (m, n)$ and $P_{m}^{n}$ being the Legandre polynomial.

\section{The study of the internal time dependence versus natural parameter of the problem - standard time}

Now we are able to turn to the prove of the possibility of the quantum chaos initiation in the wave function (10) and as a result in the probability (13). It is enough to show, that the solution $x^{1}(s)$ with certain initial conditions behaves unstable or chaotically. With that purpose on the example of elementary reaction $L i+(F H) \rightarrow(L i F H)^{*} \rightarrow(L i F)+H$ we studied carefully the behaviour of the image point trajectories on Lagrange surface $S_{p}$. It was shown that with collision energy $E_{k}^{i}=1.4 \mathrm{eV}$ and for fixed transversal vibrations energy $E_{v}=1.02 \mathrm{eV}$ the image point trajectory is stable. The whole region of kinetic energies is splited to regular subregions, and dependingly from which subregion trajectory starts it goes either to (out) channel (Fig.1(a)) or reflects back in the (in) channel (Fig.1(b)).

With a further change of kinetic energy the image point trajectory in the interaction region starts orbiting, that corresponds to the creation of the resonance complex $(\mathrm{LiFH})^{*}$, and after that leave the interaction region either to (out) (Fig.1(c)) or return to (in) channel. In such a case the image point trajectories diverge and this divergence is exponential, as can be seen from the study of the Lyapunov parameters. That is for those initial conditions the evolution in the correspondent classical problem is chaotic and so the motion of the local coordinate system is chaotic too. It is easy to see that in such situation the behaviour of $x^{1}(s)$ is also chaotic and the same is true for internal time, that is the natural parameter of quantum evolution problem.

It can be shown, that chaotic behaviour of the internal time is followed by the stochastic behaviour of the model equation solution $\xi(\tau(s))$ and the same is true for the wave function (10) and transition probability (13). In such a way the possibility of violation of quantum determinism and quantum chaos organization was shown on the example of the wave function of the simple model of multichannel scattering. 
a)

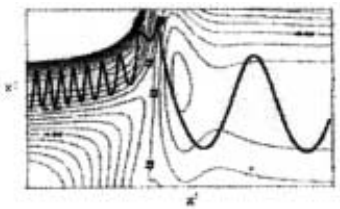

b)

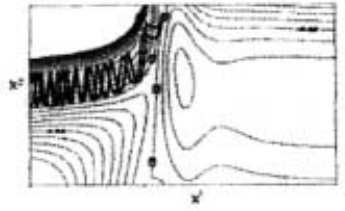

c)

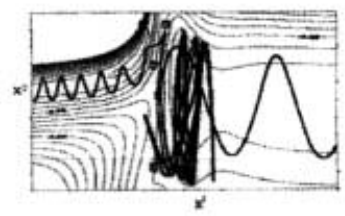

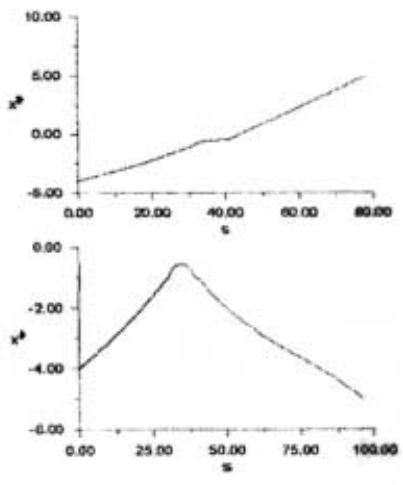

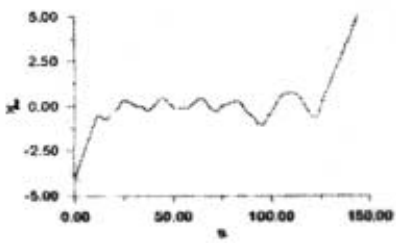

Fig. 1. Dependence of Lyapunov exponent over time parameter $s$ for the case of rearrangement reaction going through resonance state.

Those results may seem strange if we take into account, that original problem (i.e. Schrödinger equation with asymptotic scattering conditions) was quite deterministic (i.e. was good candidate for possessing unique solution), outside of standard interpretation of quantum mechanical quantities. At the same time if one looks carefully at the final version of the scattering probabilities it is clear, that difference between stochastic and regular regimes are not of principal importance, actually the ansatz of solution in our approach for two cases is the same. The only difference comes from the fact, that when orbiting in interaction region starts, the initial conditions for outcoming bunch of trajectories become undetermined, that can be regarded in terms of fluctuations of the initial stratificated Lagrange surface $S_{p}$,just as in the case of vacuum fluctuations in quantum field theory [5].

\section{Conclusion}

In this work it was shown that the representation developed by the authors includes not only Plank's constant $\hbar$, but new energetic parameter as well. Thus, when the energy of the particles collision exceeds certain critical value (which is different for the different systems), solution for internal time $\tau$ coincides with an ordinary time - natural $t$ parameter. In this case, the movement equation for the system of bodies transforms to common nonstationary Schrödinger's equation. The scattering process is in fact a direct process for this case.

But everything is quite different when the collision occurs below the critical energy specified. As it is shown, in such a case the solution for internal time $\tau$ in 
a definite range of $t$ has an oscillational character. Moreover, for all the extreme points the derivative of $\tau$ by $t$ has a jump of the first kind, while the phase portrait of reactive (parametric) oscillator has bifurcations. Let's note that these are the collision modes with the strong interference effects, i.e. the problem becomes essentially multichannel and includes the phase of resonant state formation. At a small decrease of collisions energy, a number of internal time oscillations grows dramatically. In this case the system loses all the information about its initial state completely. Chaos arises in a wave function, which then self-organizes into a new order within the limit $\tau \rightarrow \infty$. Mathematically it becomes possible as a result of common wave equation irreversibility by time.

Let's stress that the above result supports the transitional complex theory, developed by Eyring and Polanyi on the basis of evristic considerations, the essence of which is statistical description of chemical reactions. The amplitude of regrouping transition in three-body system is investigated in the work on example of $L i+(F H)_{n} \rightarrow(L i F)_{m}+H$ reaction and it is shown, that in the area where the number of internal time peculiarities is high, it has an accidental value. It is also shown that the representation developed satisfies the limit transitions in the areas specified, including transition from $Q_{c h}$ area into $P$ area. The latter occurs under $\hbar \rightarrow 0$ and at $E_{k}^{i}<E_{c}$, where $E_{c}$ is critical energy and $E_{k}^{i}$ is a collision energy. It is possible to give very simple interpretation of the above results in terms of initial Lagrange surface fluctuations in strong interaction region.

\section{References}

[1] M. C. Gutzwiller, Chaos in Classical and Quantum Mechanics, Springer, Berlin, 1990.

[2] E. Nelson, Phys. Rev., (1966), v. 150, p. 1079.

[3] A. V. Bogdanov, A. S. Gevorkyan, Three-body multichannel scattering as a model of irreversible quantum mechanics, Proceedings of the International Symposium on Nonlinear Theory and its Applications, Hilton Hawaiian Village, 1997, V.2, pp.693696.

[4] A. V. Bogdanov, A. S. Gevorkyan, Multichannel Scattering Closed Tree-Body System as a Example of Irreversible Quantum Mechanics, Preprint IHPCDB-2, 1997, pp. 1-20.

[5] A.V. Bogdanov, A.S. Gevorkyan, A.G. Grigoryan, Random Motion of Quantum Harmonic Oscillator. Thermodynamics of Nonrelativistic Vacuum, in Proceedings of Int. Conference "Trends in Math Physics", Tennessee, USA, October 14-17, 1998, pp.79-107.

[6] A.V. Bogdanov, A.S. Gevorkyan, Theory of Quantum Reactive Harmonic Oscillator under Brownian Motion, in Proceedings of the International Workshop on Quantum Systems, Minsk, Belarus, June 3-7, 1996, pp.26-33.

[7] A.V. Bogdanov, A.S. Gevorkyan, A.G. Grigoryan, S.A. Matveev, Internal Time Peculiarities as a Cause of Bifurcations Arising in Classical Trajectory Problem and Quantum Chaos Creation in Three-Body System, in Proceedings of Int. Symposium "Synchronization, Pattern Formation, and Spatio-Temporal Chaos in Coupled Chaotic Oscillators" Santyago de Compostela, Spain, June 7-10, 1998; 
[8] A.V. Bogdanov, A.S. Gevorkyan, A.G. Grigoryan, First principle calculations of quantum chaos in framework of random quantum reactive harmonic oscillator theory, in Proceedings of 6th Int. Conference on High Performance Computing and Networking Europe (HPCN Europe '98), Amsterdam, The Netherlands, April, 1998 